As a simple illustration of earthquake radiation, we may inagine a disturbance to originate at $\mathrm{O}$ as a single impulse, the resulting vibrations spreading in all directions through the earth, and in all directions over its surface. The former of these may be regarded as elastic vibrations, whilst the latter have the character of surface undulations influenced by gravity. At any station $\mathrm{P}_{1}$ the first arrivals would be preliminary tremors, chiefly compressional in character. These would be suddenly eclipsed by vibrations, probably distortional, originating by refraction beneath the crust in the vicinity of $P_{1}$. The first of them we should expect to find serrated, whilst their followers emerging between $P_{1}$ and $P_{2}$ would be smoother in outline and larger in amplitude. The last and largest members of the series would be those which have travelled practically as free surface-waves through the crust. The result of such radiation as exhibited on a seismogram would be to show true preliminary tremors, suddenly followed by a series of larger waves, which would gradually grow in size. If at the origin there were several impulses, then these latter precursors would arrive in groups. An alternative hypothesis is to assume that all the vibrations recorded at a station $\mathrm{P}$ arrived along their peculiar brachistochronic paths through the earth, an important fact supporting which, is that up to the present we have not with any certainty identified waves which may have reached $\mathrm{P}$ passing outwards from $\mathrm{O}$ round our world in opposite directions. Although it is not likely that I shall be able, in the tremor-haunted, damp, dark stable where I work, to catch the waves which have taken the longest route to my observing station, that there are such surface undulations radiating in all directions from an epifocal area there is but little doubr. Near to an origin you see the little waves come rolling down a street, whilst at distances of 300 miles the ground swell may be so heavy that I and many others have been seized with nausea. What proportion of seismic energy escapes round the surface of our earth, as compared with that which passes through the same, I do not know; but if the experiment were made, I should not be surprised to find that at the time of large earthquakes, mountains swayed like the masts of ships on a slowly heaving ocean.

All that has here been suggested is clearly very far from being above criticism. It indicates a want of knowledge respecting the researches of the elastician, whilst the facts are few. Although the observations may be characterised by their poverty, I often see in the rough-headed mobs of earthquake precursors rhythmical repetition; and I trust that, if my story of their creation and long duration is not the true one, it may at least induce others to attempt better hypotheses. JoHn MiLne.

\section{The Orbit of Witt DQ.}

THE extreme eccentricity of the orbit of Witt's planet suggests some interesting speculations. Assuming the aphelion and perihelion distances in terms of the earth's mean distance are respectively $\mathrm{I} 79$ and $I \cdot \mathbf{I} 2$, the planet approaches the sun in 322 days, a distance of sixty-one million miles, an average of 200,000 miles a day.

Practically this may be considered as a fall, during the halfrevolution, of this distance. Now if the planet were a perfectiy plastic body, and we knew all its elements, it would be perfectly possible to determine the deforming forces acting on it during the passage. It is evident that the force of gravity acting on the forward point of the syzygial axis would always be in excess of that on the rear, and in consequence that the tendency would be to continually lengthen that axis in a proportion referable to the squares of the distances fallen. On the other hand the force of internal gravitation towards its own centre would always tend to restore the sphericity, and the result would be that a body starting as a sphere from aphelion would find the syzygial axis prolonged and its shape deformed into an increas. ingly prolate spheroid, till on its arrival at perihelion and its commencement to retreat the reverse phenomenon would occur, and the planet on its return become again a sphere.

Now, of course, we have no reason to suspect that $D Q$ is a plastic body, and the comparative insignificance of its size, would, were it to be composed of matter of equal rigidity with ordinary rocks on the surface of the earth, enable it to successfully resist these deforming influences. We may, however, imagine a case where the strains would be sufficient to break up an ordinarily rigid body, if the eccentricity exceeded a certain amount, and the consequent differential action of gravity became sufficiently great.

$$
\text { NO. I } 53 \text { I, VOL. 59] }
$$

A hypothetical planet moving in an orbit of high eccentricity, for instance, between Mars and Jupiter might, so long as it continued plastic, preserve its condition as a single coherent body. If, however, it were cooled to an extent sufficient to become enveloped by a rigid crust, there might come a time when the deforming forces would cause deep and continually proceeding fractures. Eventually we can conceive that these fractures would split the body into fragments, each of which from its own intrinsic rigidity would be able to maintain its shape and cohesion. In such a case each of the fragments would proceed to take up an independent motion of its own. Hence, perhaps, we may see our way, without calling in any extraneous factor, to account for the present zone of asteroids, as well as explain the small size of the individuals.

This tallies, moreover, with observation. The great planets have all orbits approaching a circle ; Jupiter, the greatest of all, has, with one exception, the smallest maximum of eccentricity ; and Mercury, the smallest, has actually the greatest. The Leonids move in a still more elongated orbit, and they are amongst the smallest celestial objects with which we are acquainted. Altogether the minuteness of the planet and the eccentricity of the orbit have some connection in fact ; this connection. I cannot believe to be fortuitous, and it seems not altogether presumptuous to refer it to a common law, which we know pervades the universe. This is my excuse for attempting to venture into a hitherto unexplored region of physics, but one pointing to vast possibilities, amongst others in geology.

Shanghai, January 17 .

Thos. W. KingSMill.

\section{The Teaching of Geometry.}

I AM sure that all mathematical teachers can thoroughly endorse Prof. Minchin's letter. The difficulty of making a change lies in the University and Civil Service examinations, which still prescribe Euclid. On the continent Euclid has been superseded by modern books, some of which might serve as a basis for a thoroughly reformed English text-book.

I am convinced that the deplorable weakness shown by almost all boys in the solution of geometrical problems, arises in great measure from Euclid; they are utterly confused by its prolixity and verbiage.

And it is not as though this prolixity meant any greater accuracy or better logical sequence. It is not proved till Book iii. that a circle can only cut a straight line in two points; but in (i. I2) this property is quietly assumer, otherwise several perpendiculars could be drawn. I. I3 simply asserts that $a+(b+c)=(a+b)+c$, but is unintelligible to beginners through its verbiage. In i. I6 we practically make an angle equal to the interior one, against the exterior angle, and then ask the pupil to see for himself that one is greater than the other, which is suspiciously like petitio principiz. In the second Book we have a number of cumbersome proofs, some of which, indeed, are now shortened to an algebraic form. (I have never been able to understand the Cambridge regulation that the sign + may be used, but not the sign -.) The Euclidean definition of proportion is quite unintelligible to beginners, while the conception of similar figures and of scale is easily grasped. To insist on young boys entering on the subtleties of the subject, is much as though one made a child beginning arithmetic read, say, the first chapter of Weber's Algebra. What is wanted is thorough ready knowledge of the properties of lines and circles. And for this I would strongly recommend practical geometry. I believe it could very easily be made a means of imparting a knowledge of geometry in its highest and widest sense.

I 5 Pemberton Gardens, N.

R. J. Dallas.

\section{American and English Winters.}

WhILE we, in the south of England, this February, have been enjoying weather of extraordinary mildness, we have read in the daily papers of bitter frost in America, and the miseries of a ferocious blizzard. It is by no means uncommon to find opposite winter weather, at the same time, east and west of the Atlantic. Can we form any exact idea as to frequency of the cccurrence?

By way of seeking light on this, I have lately compared Chicago and Greenwich weather in the first quarter of the year, in the fifty-one years $1841-91$; presenting the facts by a variety of the graphic method, which I do not remember to have seen 